\title{
TransLiG: a de novo transcriptome assembler that uses line graph iteration
}

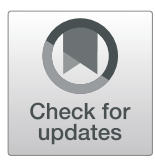

Juntao Liu', Ting $\mathrm{Yu}^{\dagger}$, Zengchao Mu and Guojun $\mathrm{Li}^{*}$

\begin{abstract}
We present TransLiG, a new de novo transcriptome assembler, which is able to integrate the sequence depth and pair-end information into the assembling procedure by phasing paths and iteratively constructing line graphs starting from splicing graphs. TransLiG is shown to be significantly superior to all the salient de novo assemblers in both accuracy and computing resources when tested on artificial and real RNA-seq data. TransLiG is freely available at https://sourceforge.net/projects/transcriptomeassembly/files/.
\end{abstract}

Keywords: RNA-seq data, Transcriptome assembly, Splicing graph, Line graph, Algorithm

\section{Background}

Alternative splicing is an important form of genetic regulation in eukaryotic genes, increasing the gene functional diversity as well as the risk of diseases [1$3]$. As reported $[4,5]$, most of the eukaryotic genes including human genes undergo the process of alternative splicing, and so one gene could produce tens or even hundreds of splicing isoforms in different cellular conditions, causing different functions and potential diseases. Therefore, the identification of all the full-length transcripts under specific conditions plays a crucial role in many subsequent biological studies. However, we are still far from a complete landscape of human transcripts, and the situation is even much less clear for non-human eukaryotic species [6].

RNA-seq is a powerful technology that enables the identification of expressed genes as well as abundance measurements at the whole transcriptome level with unprecedented accuracy [7-10]. The RNA-seq protocol takes as input the sampled expressed transcripts and produces more than 200 million short reads for a run, and each sequencing read is generally of length 50-150 base pairs, posing great challenges to reconstruct the full-length transcripts from the RNA-seq reads. Firstly, different transcripts could have highly different expression abundances, which makes the constructed sequence graph (splicing graph, de bruijn

\footnotetext{
* Correspondence: guojunsdu@gmail.com

†u

School of Mathematics, Shandong University, Jinan 250100, China
}

graph, etc.) have quite uneven coverage. Secondly, different transcripts from the same gene can share exonic sequences due to alternative splicing, making the splicing graph even more complicated. Thirdly, a large amount of RNA-seq reads contain sequencing errors, making it more difficult to assemble those lowly expressed transcripts from the RNA-seq data. All of the above have made the transcriptome assembly problem highly challenging.

There have been a growing number of methods developed for solving the transcript assembly problem in recent years, and most of them could be categorized into two approaches: the reference-based (or genome-guided) and the de novo $[11,12]$. The reference-based approaches such as Scallop [13], TransComb [14], StringTie [6], Cufflinks [15], and Scripture [16] usually first map the RNA-seq reads to a reference genome using alignment tools such as Hisat [17], Star [18], Tophat [19], SpliceMap [20], MapSplice [21], or GSNAP [22], and the reads from the same gene locus would fall into a cluster to form a splicing graph, and all the expressed transcripts could be assembled by traversing the graphs. The assembled transcripts by this strategy generally have higher accuracy compared to those by de novo strategy as it benefits from a reference genome, but it is seriously limited in practice because such a high-quality reference genome is currently unavailable for most species.

(c) The Author(s). 2019 Open Access This article is distributed under the terms of the Creative Commons Attribution 4.0 International License (http://creativecommons.org/licenses/by/4.0/), which permits unrestricted use, distribution, and reproduction in any medium, provided you give appropriate credit to the original author(s) and the source, provide a link to the Creative Commons license, and indicate if changes were made. The Creative Commons Public Domain Dedication waiver (http://creativecommons.org/publicdomain/zero/1.0/) applies to the data made available in this article, unless otherwise stated. 
De novo assembly is a desired approach when the reference genome is unavailable, incomplete, highly fragmented, or substantially altered as in cancer tissues. There have been a number of de novo assemblers, such as BinPacker [23], Bridger [24], Trinity [12], IDBA-Tran [25], SOAPdenovo-trans [26], ABySS [27], and Oases [28]. This strategy usually directly constructs splicing graphs from RNA-seq reads based on their sequence overlaps, and then assembles transcripts by traversing the graphs using different algorithms. Assemblers like IDBA-Tran, SOAPdenovotrans, ABySS, and Oases were developed based on the key techniques in genome assembly, and so in general, they do not work well in transcriptome assembly. Trinity opens the door to design a method specifically for handling the de novo assembly of transcriptome. It first extends the sequencing reads into long contigs by a k-mer extension strategy, then connects those contigs into a de bruijn graph, and finally infers all the expressed transcripts by traversing the de bruijn graph. As noticed in the Trinity paper, there are some limitations hindering its applications. The sequence depth information which would be useful in the assembling procedure was not adequately used, and a brute force strategy was applied to search for transcript-representing paths in the de bruijn graph, causing it to suffer seriously from false positive rates. Bridger successfully transplants the minimum path-cover model from the reference-based assembler Cufflinks to the de novo assembly and effectively avoids the exhaustive enumeration, making the false positives highly decreased. However, it does not make full use of the sequence depth information which should be useful in the development of assembling procedure as mentioned in the Trinity paper. Subsequently, a new assembler BinPacker was developed to fully use the sequence depth information by a bin-packing model without limiting the minimum number of paths. BinPacker performs better than others of same kind, but it has not integrated the paired-end information into the assembling procedure, leaving a big room to be improved.

In this paper, we introduce a new de novo assembler TransLiG developed by phasing paths and iteratively constructing weighted line graphs starting from splicing graphs. The idea of phasing paths in TransLiG was motivated from Scallop [13], a reference-based transcriptome assembler, which also adopted a similar strategy of phasing paths in a graph. Although Scallop and TransLiG shared the same idea of graph decomposition, they were differently using the sequence depth and paired-end information. Different from Scallop which decomposed graphs by iteratively constructing local bipartite graphs, TransLiG pursued the globally optimum solution by iteratively building weighted line graphs.

TransLiG was developed to integrate the sequence depth and pair-end information into the assembling procedure by phasing paths and iteratively constructing line graphs, making it substantially superior to all the salient tools of same kind, e.g., Trinity, Bridger, and BinPacker. When tested on both the artificial and real data, it reaches the precision 6\% higher than BinPacker and Bridger, and nearly $15 \%$ higher than Trinity on artificial data, and 7\%, 14\%, and 21\% higher than BinPacker, Bridger, and Trinity respectively, on the tested mouse data. Not only does TransLiG achieve the highest precision, but also it reaches the highest sensitivity on all the tested datasets. In addition, TransLiG stably keeps the best performance with different assessment parameters.

\section{Results and discussion}

We compared TransLiG with five salient de novo assemblers: BinPacker (version 1.0), Bridger (version r2014-12-01), Trinity (version 13.02.25), IDBA-Tran (version 1.1.1), and SOAPdenovo-trans (version 1.0.3) on both artificial and real datasets. The parameter settings for each of them are described in the Additional file 1: Supplementary Notes.

\section{Assessment metrics and performance evaluation}

Commonly used criteria were applied to the evaluation of all the salient de novo assembling algorithms in this experiment. Assembled transcripts by each assembler were aligned to the expressed transcripts in ground truth using BLAT [29] with 95\% sequence identity as cutoff. An expressed transcript is called full-length reconstructed if it was covered by an assembled transcript with at least $95 \%$ sequence identity and no more than $5 \%$ insertions or deletions (indels), and this assembled transcript is called a true positive. To make more convincing comparisons, we also considered different sequence identity levels $(90 \%, 85 \%$, and $80 \%)$ to define full-length reconstructed reference transcripts and true positives. Accuracy is measured by sensitivity and precision, where sensitivity is defined as the number of full-length reconstructed transcripts in ground truth by an assembler, and precision is defined as the fraction of true positives out of all assembled transcripts.

\section{Evaluation on simulation data}

We first tested TransLiG against the other assemblers on the simulation data which was generated by the tool Flux Simulator [30] using all the known human transcripts (approximately 83,000 sequences) from the UCSC hg19 gene annotation. The generated dataset contains approximately 55 million strand-specific RNA-seq paired-end reads of 76-bp length. 
Testing all the six assemblers on the simulation data, we found that TransLiG reconstructed 7935 full-length expressed transcripts, while BinPacker, Bridger, Trinity, IDBA-Tran, and SOAPdenovo-trans respectively recovered $7602,7572,6863,5405$, and 7080 full-length expressed transcripts. Therefore, TransLiG reaches the highest sensitivity followed by BinPacker and Bridger. The reason why BinPacker and Bridger are inferior to TransLiG while superior to the others is simply because they employed appropriately mathematical models in their assembly procedures, while they did not sufficiently use the paired-end and sequence depth information. Trinity was brutally enumerating all the paths over de Bruijn graphs, and thus generating a large amount of transcript candidates. However, the brute force strategy involves too many false positives, leading to a poor sensitivity. IDBA-Tran behaves the worst among all the compared tools. We can see from Fig. 1a that TransLiG recovered $4.38 \%$ more full-length expressed transcripts than the next best assembler BinPacker, and $15.62 \%$ more than Trinity. In addition, TransLiG consistently behaves the best in sensitivity under different sequence identity levels (95\%, 90\%, 85\%, and $80 \%)$ as illustrated in Fig. 1a and Additional file 1: Table S1. Not only does TransLiG perform better in sensitivity than all the compared tools, but also it does in precision. It reaches the highest precision of $44.21 \%$ versus BinPacker of $38.01 \%$, Bridger of $37.94 \%$, Trinity of $29.43 \%$, IDBA-Tran of $27.33 \%$, and SOAPdenovo-trans of $27.37 \%$, and keeps its superiority under different sequence identity levels (see Fig. 1b and Additional file 1: Table S1). SOAPdenovo-trans recovered more full-length expressed transcripts than
Trinity, but its precision is the lowest among all the compared tools.

We also compared their abilities of reconstructing expressed full-length transcripts at different abundance levels, which was evaluated by recall defined as the fraction of full-length reconstructed expressed transcripts out of all expressed transcripts under different transcript abundance levels. We can see from Fig. 1c that IDBA-Tran shows the lowest recall under all the abundance levels, and the recalls of Trinity under low abundances (1-10 and 10-20) are similar with BinPacker and Bridger, a little higher than SOAPdenovo-trans. However, SOAPdenovo-trans demonstrates much higher recalls than Trinity under high abundances (20-100). TransLiG is consistently superior to all the compared tools in recall across all the expression levels.

We further compared the performance of the assemblers in identifying expressed genes. A gene is considered to be correctly identified if at least one of its transcripts was correctly assembled. We found that TransLiG correctly identified 6189 genes, while BinPacker, Bridger, Trinity, IDBA-Tran, and SOAPdenovo-trans identified 5984, 5979, 5247, 4865, and 5951, respectively. Therefore, TransLiG outperforms all the compared assemblers in terms of the number of expressed genes identified.

\section{Evaluation on real data}

In this section, we tested the six assemblers on the following three real biological datasets, the human $\mathrm{K} 562$ cells, the human $\mathrm{H} 1$ cells, and the mouse dendritic cells datasets, containing 88 million, 41 million, and 53 million paired-end reads, respectively. They were collected from the NCBI Sequence Read Archive (SRA) database
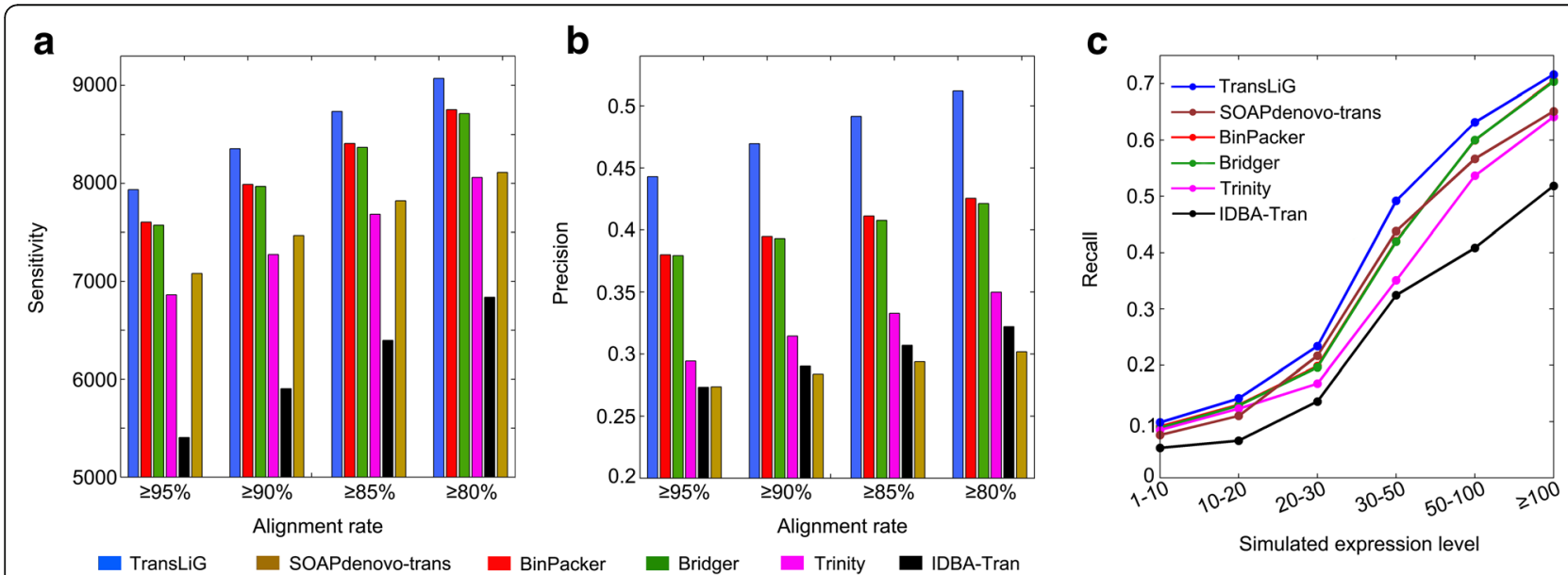

Fig. 1 Comparison results on simulated data. a Comparison of sensitivity distributions of the six tools against different sequence identity levels. $\mathbf{b}$ Comparison of precision distributions of the six tools against different sequence identity levels. c Comparison of recall distributions of the six tools against transcript expression levels 
with accession codes SRX110318, SRX082572, and SRX062280, respectively. Different from simulated datasets, which provide us the explicit ground truth, it is impossible for us to know all the genuine transcripts encoded in the real datasets. Despite this, we collected all the currently known transcripts in the UCSC genome databases as references. The versions of human and mouse reference transcripts used in this study are GRCh37/hg19 and GRCm38/mm10, respectively.

Running the six assembling tools on the human K562 cells, the human $\mathrm{H} 1$ cells and the mouse dendritic cells datasets, we found that TransLiG recovered 9826, 10,017, and 12,247 full-length reference transcripts respectively on the three real datasets, versus 9454,9557 , and 11,761 by the second best assembler BinPacker, and 8315, 8516, and 9937 by Trinity, i.e., TransLiG recovered $3.93 \%, 4.81 \%$, and $4.13 \%$ more full-length reference transcripts than BinPacker, and $18.17 \%, 17.63 \%$, and $23.25 \%$ more than Trinity. In addition, TransLiG consistently keeps the highest sensitivity under different sequence identity levels (see Fig. 2 and Additional file 1: Table S2-S4), clearly indicating its higher reliability and stability.

As for precision, TransLiG reaches $12.90 \%, 12.72 \%$, and $33.26 \%$ on the human K562 cells, the human H1 cells and the mouse dendritic cells datasets, respectively, versus $10.18 \%, 8.57 \%$, and $26.03 \%$ by the second best BinPacker, and $7.24 \%, 4.92 \%$, and $12.37 \%$ by Trinity. Similar to the results on the simulation datasets, SOAPdenovo-trans shows the lowest precision among all the compared tools on the real datasets. By comparisons, we see that TransLiG has been significantly improved in precision compared to the others, especially on the mouse data, where the TransLiG achieves 7\% more than the next best BinPacker, and 21\% more than
Trinity. In addition, the superiority of TransLiG was also clearly demonstrated by changing the sequence identity levels (see Fig. 3 and Additional file 1: Table S2-S4).

Similarly, we also compared the assemblers in terms of the numbers of the expressed genes identified on real datasets. We found that TransLiG correctly identified 5468,5669 , and 7425 genes on the human K562 cells, the human $\mathrm{H} 1$ cells, and the mouse dendritic cells datasets, respectively, versus 5359, 5485, and 7250 by BinPacker; 5330,5509 , and 7284 by Bridger; 4726, 5003, and 6262 by Trinity; 2823, 3387, and 4533 by IDBA-Tran; and 4179 , 4403, and 7145 by SOAPdenovo-trans.

The evaluations on both artificial and real datasets have fully demonstrated that TransLiG consistently shows the best performance among all the salient tools of same kind no matter in terms of sensitivity, precision, or the number of identified genes.

\section{Evaluation of computing resource usage}

De novo assemblers generally consume large computing resources (e.g., CPU time and memory usage). We by Fig. 4 and Fig. 5 illustrate the $\mathrm{CPU}$ time and memory (RAM) usage by individual assemblers on the real datasets. We see from Fig. 4 that Trinity and IDBA-Tran consume much more CPU times than all the others on all the three datasets. SOAPdenovo-trans is the fastest one among all the compared tools, while TransLiG, BinPacker, and Bridger cost CPU times similar to SOAPdenovo-trans. As for the RAM usage, Fig. 5 shows that Trinity consumes much higher memory than all the others on all the three datasets, where TransLiG, BinPacker, and Bridger cost similar memory resources, but higher than IDBA-Tran and SOAPdenovo-trans. IDBA-Tran is the most parsimonious one followed by SOAPdenovo-trans in terms of RAM usage. Overall,

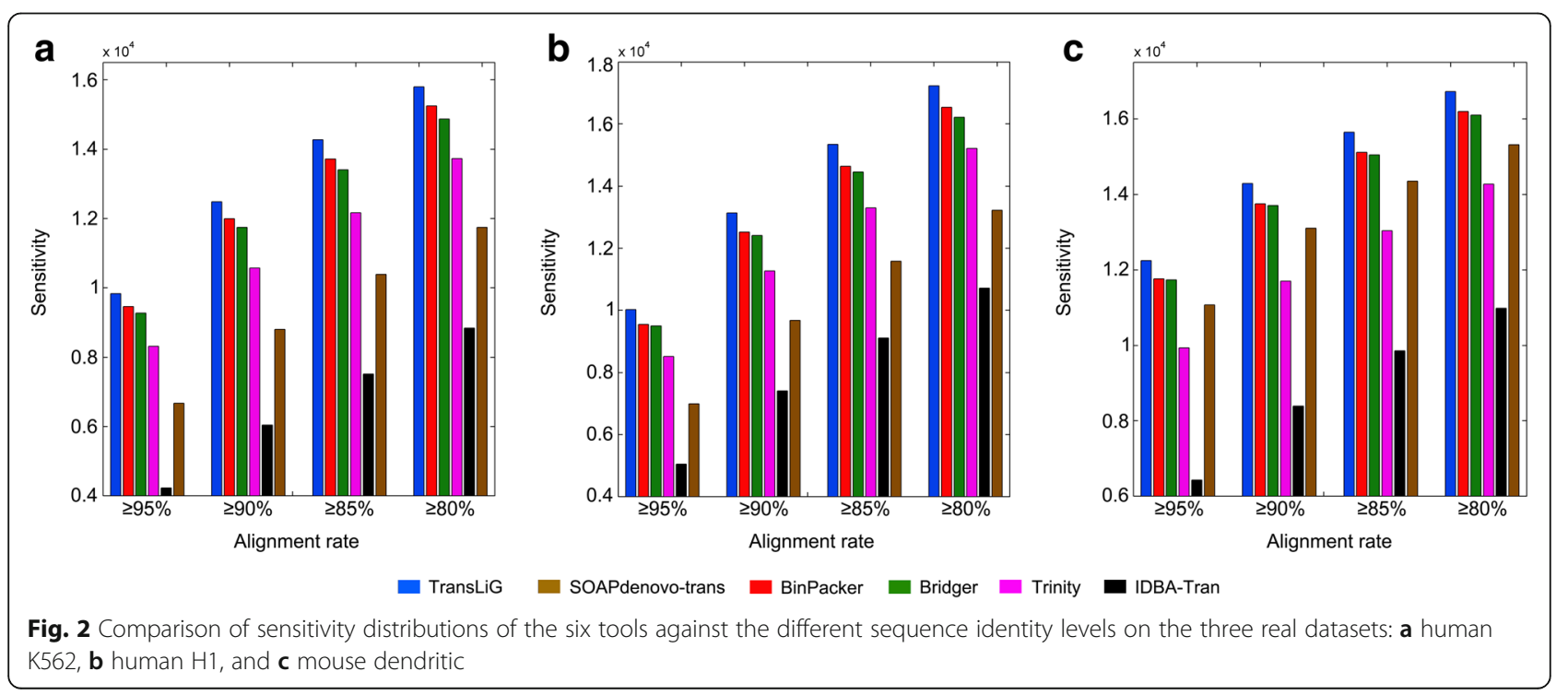




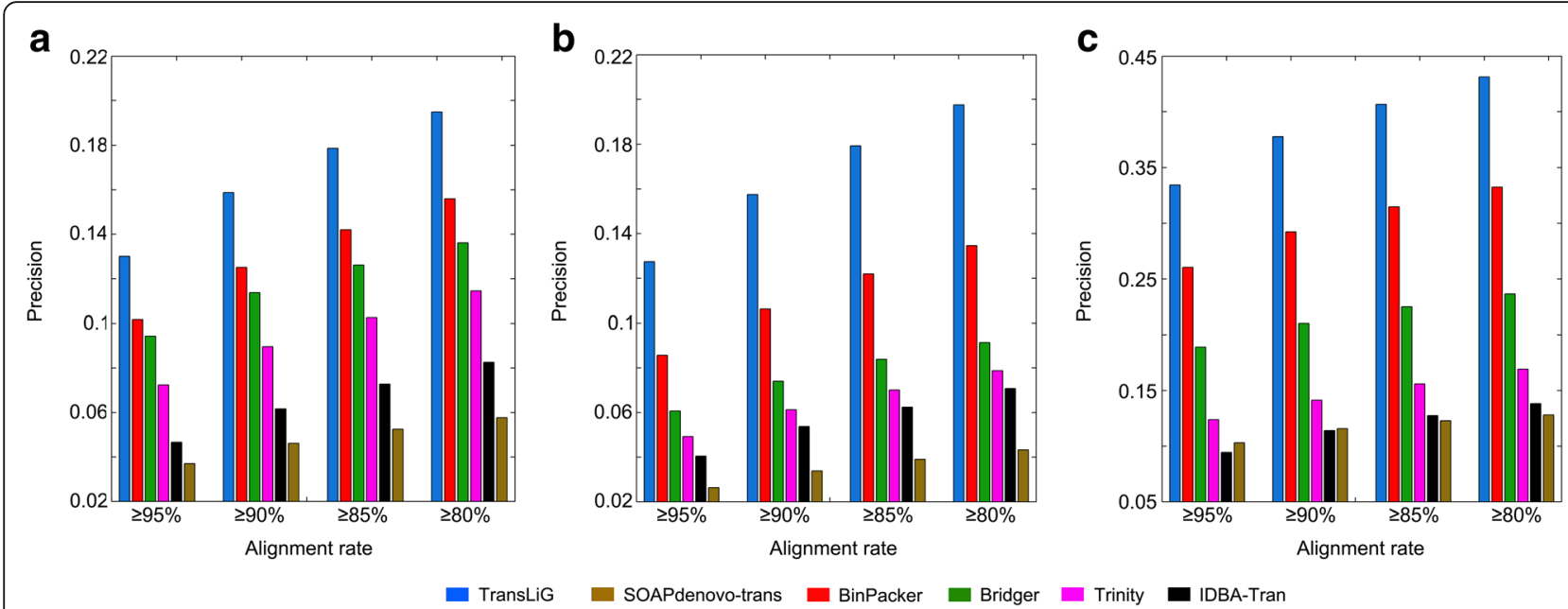

Fig. 3 Comparison of precision distributions of the six tools against different sequence identity levels on the three real datasets: $\mathbf{a}$ human $\mathrm{K} 562$, $\mathbf{b}$ human $\mathrm{H} 1$, and $\mathbf{c}$ mouse dendritic

TransLiG is not the most parsimonious, but it is quite acceptable for practical use.

\section{Conclusions}

In this study, we presented a novel de novo assembler TransLiG for transcriptome assembly using short RNA-seq reads. Compared to the salient assemblers of same kind on both simulated and real datasets, TransLiG consistently performs the best in accuracy (including sensitivity and precision) and the number of correctly identified genes. The superiority may attribute to the following facts. Firstly, TransLiG constructs more accurate splicing graphs by reconnecting fragmented graphs via iterating different lengths of smaller k-mers. Secondly, TransLiG substantially integrates the sequence depth and paired-end information into the assembling procedure via enforcing each pair-supporting path being included in at least one assembled transcript. Thirdly, TransLiG accurately links the in-coming and out-going edges at each node via iteratively solving a series of quadratic programmings, which are optimizing the utilizations of the paired-end and sequencing depth information. Finally, TransLiG benefits from the iterations of weighted line graphs constructed by repeatedly phasing transcript-segment-representing paths. Notice that the final line graph $L^{n}(G)$ is empty, and the isolated nodes generated during the line graph iteration could be expanded into assembled transcripts.

To our best knowledge, TransLiG is the first de novo assembler which effectively integrates the paired-end and sequence depth information into the assembling procedure via phasing and contracting paths with the help of line graph iterations. The software has been developed to be user-friendly and expected to play a

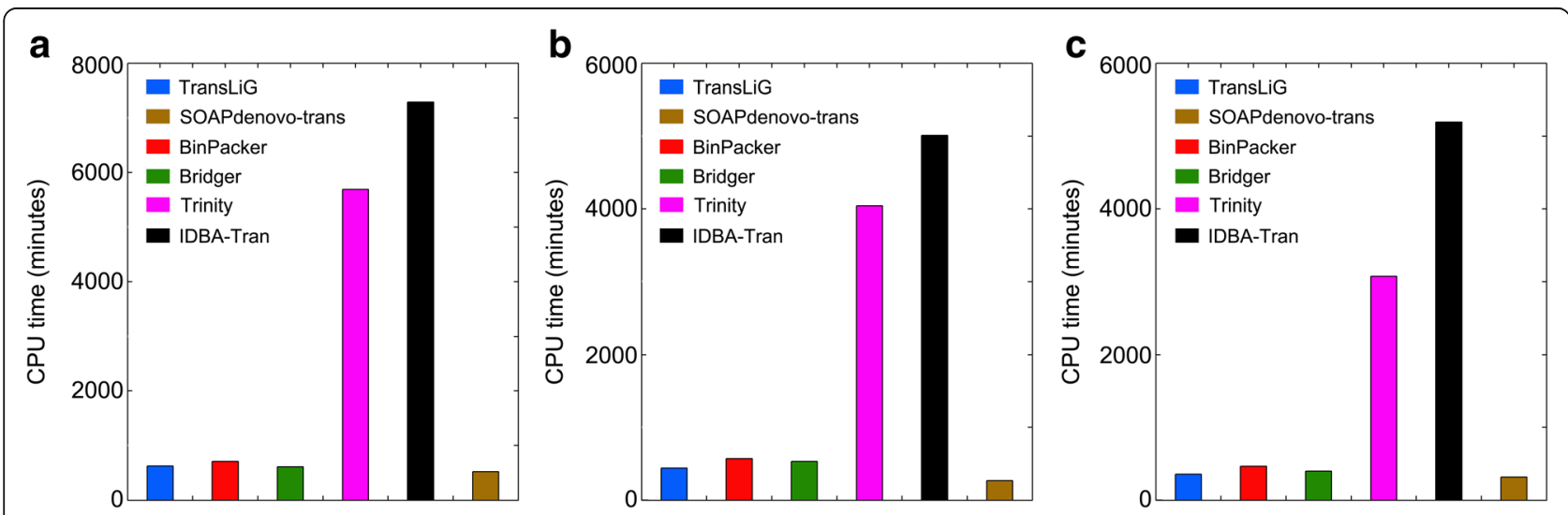

Fig. 4 Comparison of CPU times for the six tools on the three datasets: a human $\mathrm{K} 562$, b human $\mathrm{H} 1$, and $\mathbf{c}$ mouse dendritic 

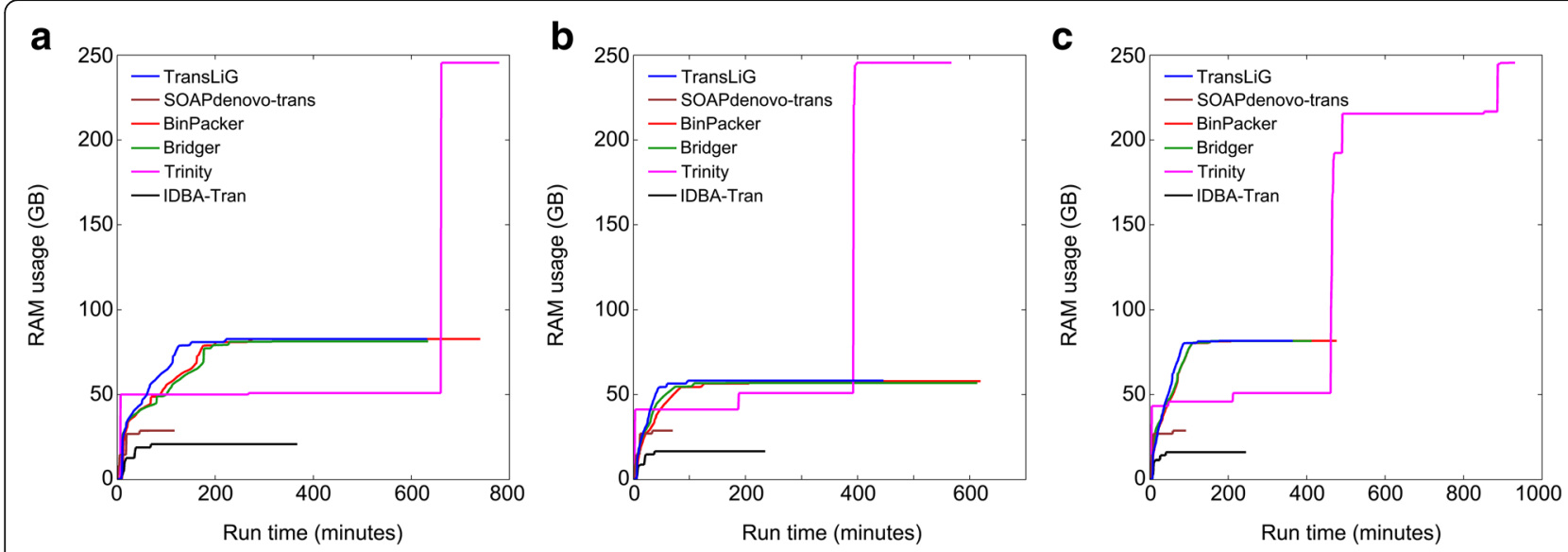

Fig. 5 Comparison of RAM usages for the six tools on the three datasets: a human K562, b human H1, and c mouse dendritic

crucial role in new discoveries of transcriptome studies using RNA-seq data, especially in the research areas of complicated human diseases such as cancers, discoveries of new species, and so on.

\section{Methods}

We designed the new de novo assembler TransLiG to retrieve all the transcript-representing paths in splicing graphs by phasing paths in the splicing graphs and iteratively constructing line graphs starting from the splicing graphs. For a graph $G=(V, E)$, the line graph $L(G)$ of $G$ is the graph with nodes representing edges of $G$, and edges representing incident relationship between edges in $G$, i.e., two nodes $u, v$ of $L(G)$, that are edges of $G$, are connected by an edge in $L(G)$ if and only if they share a node in $G$. Obviously, the line graph of a directed acyclic graph (DAG) remains a DAG. Therefore, the line graph of a splicing graph must be a DAG. By $L^{n}(G)$, we define the line graph of $G$ of order $n$, i.e., $L^{n}(G)=L\left(L^{n-1}(G)\right.$ ), where $L^{0}(G)=G$. It then turns out that $L^{n}(G)$ is an empty graph, i.e., a graph with all nodes being isolated for a DAG graph $G$ of $n$ nodes. Obviously, each isolated node generated during the line graph iteration can be expanded into a transcript-representing path $P$ in $G$.

The basic idea behind TransLiG is to globally optimize the accuracy of retrieving all the full-length transcripts encoded in a splicing graph by phasing paths and iteratively constructing the next line graph $L^{i+1}(G)$ weighted by solving series of quadratic programming problems defined on the current (line) graph $L^{i}(G)$. After removing all the zero-weighted edges from the constructed line graph, the remaining graph ideally consists of the line graph edges of individual transcript-representing paths in the current graph $L^{i}(G)$. Continuing the iteration until $L^{n}(G)$ becomes empty. Hopefully, each isolated node generated during the line graph iteration will be expanded into a transcript-representing path, which exactly corresponds to an expressed transcript. The flowchart of the TransLiG is roughly outlined in Fig. 6 and followed by the pseudo-codes of TransLiG.

\section{De novo assembling algorithm TransLiG \\ Step 1: Construction of splicing graphs}

We first construct initial splicing graphs based on the graph-building framework of BinPacker [23], and then, we designed a novel technique to effectively modify the initial splicing graphs by merging the isolated pieces (see Additional file 1: Methods 2.1 for details).

\section{Step 2: Phasing pair-supporting paths}

Ideally, each expressed transcript $T$ corresponds to a unique path $P_{T}$ in the splicing graph, and so a segment in the transcript $T$ corresponds to a sub-path of $P_{T}$, which is called a transcript-segment-representing path later on. Two paired-end reads $r_{1}$ and $r_{2}$ are supposed to come from a single transcript, corresponding to a segment in the transcript, and so a transcript-segment-representing path in the splicing graph. The transcript-segment-representing path in this paper is called a pair-supporting path. TransLiG retrieves a set, denoted by $P_{G}$, of all the pair-supporting paths for each splicing graph $G$.

In detail, for each single-end read $r$, if it spans a sub-path $P=n_{i 1} \rightarrow n_{i 2} \rightarrow \ldots \rightarrow n_{i p}$ of graph $G$ and $p \geq 3$, then we add a pair-supporting path $P=n_{i 1} \rightarrow n_{i 2} \rightarrow$ $\ldots \rightarrow n_{i p}$ to $P_{G}$. For the case of paired-end reads $r_{1}$ and $r_{2}$, if $r_{1}$ spans a sub-path $P_{1}=n_{i 1} \rightarrow n_{i 2} \rightarrow \ldots \rightarrow n_{i p}$, and $r_{2}$ spans a sub-path $P_{2}=n_{j 1} \rightarrow n_{j 2} \rightarrow \ldots \rightarrow n_{j q}$, and there exists a unique path $P_{i n}=n_{i p} \rightarrow n_{m 1} \rightarrow n_{m 2} \rightarrow \ldots \rightarrow$ $n_{m k} \rightarrow n_{\mathrm{j} 1}$ between $n_{i p}$ and $n_{j 1}$ in $G$, and $p+k+q \geq 3$, we then add a pair-supporting path $P=P_{1} \rightarrow P_{\text {in }} \rightarrow P_{2}$ to $P_{G}$. Different reads (single-end or paired-end) may generate the same pair-supporting path. So, each pair-supporting 


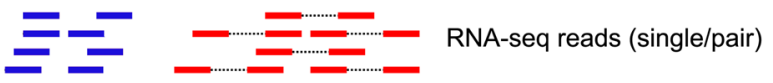

Step 1. Construction of splicing graphs

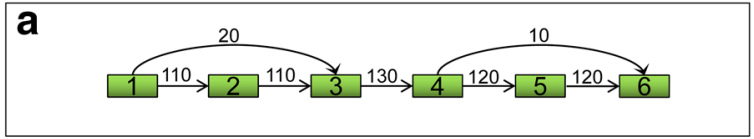

Step 2. Phasing pair-supporting paths

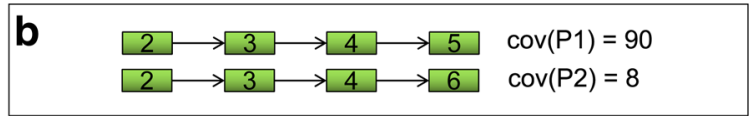

Iteration 1 Step 3. Iteratively construction of weighted line graphs

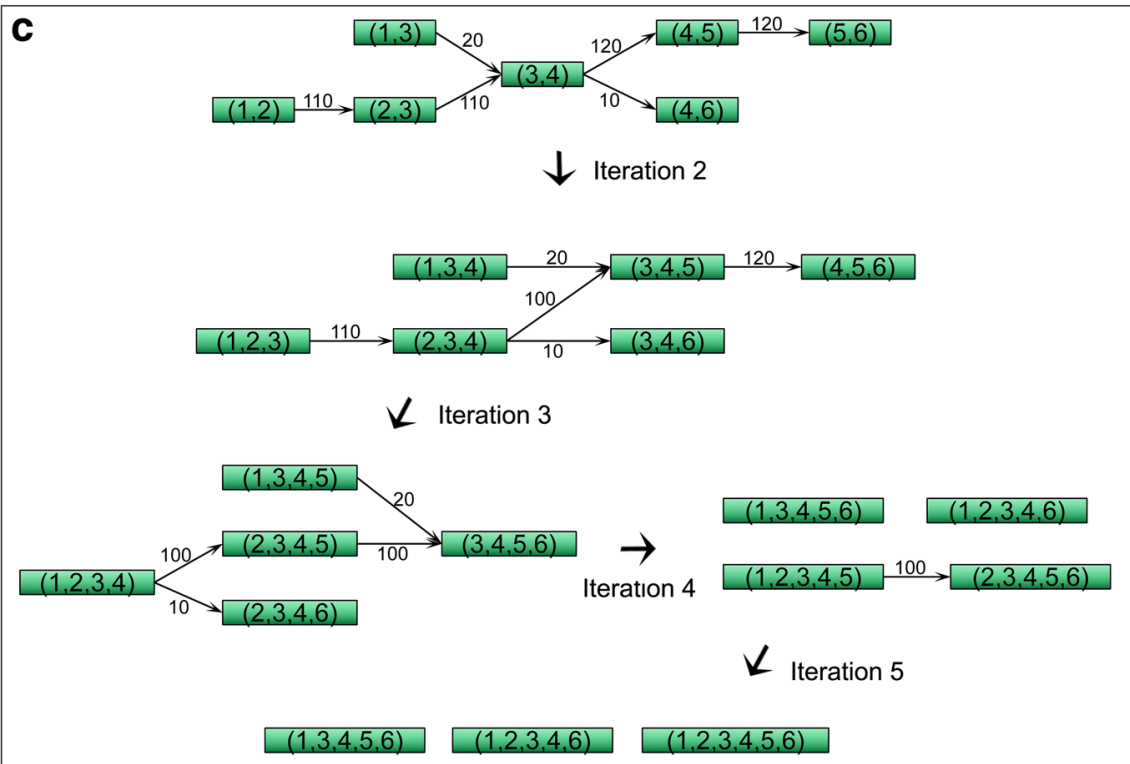

Step 4. Recovery of transcripts

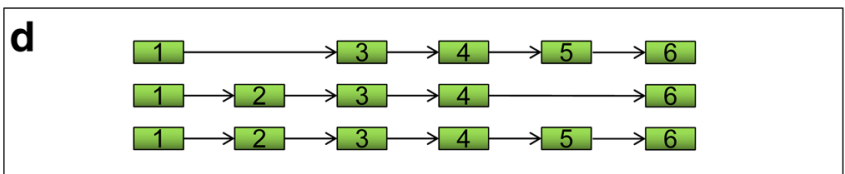

Fig. 6 Flowchart of TransLiG. a TransLiG takes as input the RNA-seq reads (single or pair) to construct splicing graphs by first using the graphbuilding framework of BinPacker to build initial splicing graphs, and then modifying initial splicing graphs by merging the isolated pieces in a specific way. b TransLiG phases pair-supporting paths from the splicing graphs to ensure that each pair-supporting path is covered by an assembled transcript. c TransLiG assembles transcripts by iteratively constructing weighted line graphs until empty. $\mathbf{d}$ The transcript-representing paths are obtained by expanding all the isolated nodes generated during the line graph iteration

path $P$ is assigned a weight $\operatorname{cov}(P)$ as the number of reads that could generate the path $P$.

\section{Step 3: Iteratively construction of weighted line graphs} Starting from a splicing graph, let $G$ be the current weighted (line) graph. TransLiG weights the line graph
$L(G)$ of $G$ via solving a quadratic program at each node of $G$. Assume that node $v$ in $G$ has $n$ in-coming edges and $m$ out-going edges. In theory, there are $m \times n$ feasible connections between the $n$ in-coming edges and $m$ out-going edges. We expect to find the correct connections that the to-be-assembled transcripts pass through. 
To achieve this goal, we designed the following programming, in which $\left(s_{j}-\sum_{j=1, \ldots, m} w_{i j} x_{i j}\right)^{2}$ measures the deviation between the weight of the in-coming edge $e_{i}$ and the sum of the weights of all the transcript-representing paths passing through $e_{i}$, and similarly for $\left(\mathrm{c}_{j}-\sum_{i=1, \ldots, n} w_{i j} x_{i j}\right)^{2}$, where $s_{i}$ is the weight of the in-coming edge $e_{i}$ at $v, c_{j}$ the weight of the out-going edge $e_{j}$ at $v ; x_{i j}$ represents a binary variable with $x_{i j}=1$ if there is at least one transcript-representing path passing through $e_{i}$ and $e_{j}$, and 0 otherwise, and $w_{i j}$ represents the coverage value of all the transcript-representing paths passing through $e_{i}$ and $e_{j}$. We then minimize the deviations for all the in-coming and out-going edges to find the correct connections between the in-coming and out-going edges. Therefore, the correct way of all the transcript-representing paths passing through the node $v$ could be determined by solving the following quadratic program.

$$
\begin{gathered}
\min z=\sum_{i=1, \ldots, n}\left(s_{i}-\sum_{j=1, \ldots, m} w_{i j} x_{i j}\right)^{2}+\sum_{j=1, \ldots, m}\left(c_{j}-\sum_{i=1, \ldots, n} w_{i j} x_{i j}\right)^{2} \\
\text { s.t. }\left\{\begin{array}{c}
w_{i j} \geq \sum_{P \in P_{G},\left(e_{i}, e_{j}\right) \subset P} \operatorname{cov}(P), \quad i=1, \ldots, n, \quad j=1, \ldots, m \\
\sum_{i=1, \ldots, n} x_{i j} \geq 1, \quad j=1, \ldots, m \\
\sum_{j=1, \ldots, m} x_{i j} \geq 1, \quad i=1, \ldots, n \\
\sum_{i j} w_{i j} \geq 0 \\
i=1, \ldots, n \\
j=1, \ldots, m
\end{array}\right.
\end{gathered}
$$

where $P_{G}$ is the set of pair-supporting paths, and $\operatorname{cov}(P)$ is the coverage of pair-supporting path $P ; M$ represents the expected number of transcript-representing paths passing through the node $v$, and clearly $\max \{m, n\} \leq$ $M \leq m \times n$ (see Additional file 1: Methods 2.2 in details for the determination of $M$ ). Clearly, this is a mixed integer quadratic programming, an NP-hard problem. However, it is computationally acceptable in our assembly procedure due to the specific properties of the constructed splicing graphs (see Additional file 1: Methods 2.3 in details for the solution of the quadratic programming).

Let $\left\{x_{i j}, w_{i j}\right\}$ be the optimum solution of the quadratic program. Then the line graph $L(G)$ of the current (line) graph $G$ is weighted by assigning $w_{i j}$ to $\left(e_{i}, e_{j}\right)$ if $x_{i j}=1$, and 0 otherwise. Reset $G$ to be the graph $L(G)$ by removing all the zero-weighted edges, and modifying the pair-supporting paths in $P_{G}$ accordingly. Then TransLiG repeats Step 3 until $L(G)$ is empty.

\section{Step 4: Recovery of transcripts}

TransLiG recovers all the transcripts by expanding all the isolated nodes generated during the line graph iteration, i.e., by tracking back to recover all the transcript-representing paths in the original splicing graphs.

\section{Additional file}

Additional file 1: This file contains the parameter setups of the compared assemblers, the supplementary methods, figures and tables. (PDF 368 kb)

\section{Abbreviations}

NCBI: National Center for Biotechnology Information; RNA-seq: RNA sequencing; SRA: Sequence read archive

\section{Acknowledgements}

Not applicable.

\section{Funding}

This work was supported by the National Natural Science Foundation of China (61801265, 61432010, 61771009), the National Key Research and Development Program of China (2016YFB0201702), the Natural Science Foundation of Shandong Province with code ZR2018PA001, and the China Postdoctoral Science Foundation with code 2018 M632659. The funders had no role in study design, data collection and analysis, decision to publish, or preparation of the manuscript.

\section{Availability of data and materials}

The TransLiG package is available at https://sourceforge.net/projects/ transcriptomeassembly/files/ [31] and https://doi.org/10.5281/zenodo. 2576226 [32] under the GNU General Public License (GPL). The three real datasets used in this study, the human $\mathrm{K} 562$ cells, the human $\mathrm{H} 1$ cells, and the mouse dendritic cells datasets can be downloaded from the NCBI Sequence Read Archive (SRA) database with accession codes SRX110318, SRX082572 and SRX062280, respectively [33]. Simulated data used in this study is available at https://sourceforge.net/projects/transassembly/files/ TransLiG-Simulation-Data/ [34].

\section{Authors' contributions}

GL conceived and designed the experiments. $J$, TY, and ZM performed the experiments. $J \mathrm{~L}$ and TY analyzed the data. TY and $J \mathrm{~L}$ contributed reagents/ materials/analysis tools. $G L$ and $J L$ wrote the paper. TY and $J L$ designed the software used in analysis. GL oversaw the project. All authors read and approved the final manuscript.

Ethics approval and consent to participate Not applicable.

\section{Consent for publication}

Not applicable.

\section{Competing interests}

The authors declare that they have no competing interests.

\section{Publisher's Note}

Springer Nature remains neutral with regard to jurisdictional claims in published maps and institutional affiliations.

Received: 23 November 2018 Accepted: 9 April 2019

Published online: 23 April 2019

\section{References}

1. Kelemen $\mathrm{O}$, Convertini $\mathrm{P}$, Zhang ZY, Wen Y, Shen ML, Falaleeva M, Stamm S. Function of alternative splicing. Gene. 2013;514:1-30. 
2. Tazi J, Bakkour N, Stamm S. Alternative splicing and disease. Biochimica Et Biophysica Acta-Mol Basis Dis. 2009;1792:14-26.

3. Singh RK, Cooper TA. Pre-mRNA splicing in disease and therapeutics. Trends Mol Med. 2012;18:472-82.

4. Wang ET, Sandberg R, Luo S, Khrebtukova I, Zhang L, Mayr C, Kingsmore SF, Schroth GP, Burge CB. Alternative isoform regulation in human tissue transcriptomes. Nature. 2008;456:470-6.

5. Pan Q, Shai O, Lee LJ, Frey J, Blencowe BJ. Deep surveying of alternative splicing complexity in the human transcriptome by high-throughput sequencing. Nat Genet. 2008;40:1413-5.

6. Pertea M, Pertea GM, Antonescu CM, Chang TC, Mendell JT, Salzberg SL. StringTie enables improved reconstruction of a transcriptome from RNA-seq reads. Nat Biotechnol. 2015;33:290-5.

7. Ozsolak F, Milos PM. RNA sequencing: advances, challenges and opportunities. Nat Rev Genet. 2010;12:87-98.

8. Wang Z, Gerstein M, Snyder M. RNA-Seq: a revolutionary tool for transcriptomics. Nat Rev Genet. 2009;10:57-63.

9. Marguerat S, Bähler J. RNA-seq: from technology to biology. Cell Mol Life Sci. 2010;67:569-79.

10. Wilhelm BT, Landry J-R. RNA-Seq-quantitative measurement of expression through massively parallel RNA-sequencing. Methods. 2009:48:249-57.

11. Martin JA, Wang Z. Next-generation transcriptome assembly. Nat Rev Genet. 2011:12:671-82.

12. Grabherr MG, Haas BJ, Yassour M, Levin JZ, Thompson DA, Amit I, Adiconis X, Fan L, Raychowdhury R, Zeng Q. Full-length transcriptome assembly from RNA-Seq data without a reference genome. Nat Biotechnol. 2011;29:644-52.

13. Mingfu Shao CK. Accurate assembly of transcripts through phase-preserving graph decomposition. Nat Biotechnol. 2017;35:1167-9.

14. Liu J, Yu T, Jiang T, Li G. TransComb: genome-guided transcriptome assembly via combing junctions in splicing graphs. Genome Biol. 2016;17:213.

15. Trapnell C, Williams BA, Pertea G, Mortazavi A, Kwan G, van Baren MJ, Salzberg SL, Wold BJ, Pachter L. Transcript assembly and quantification by RNA-Seq reveals unannotated transcripts and isoform switching during cell differentiation. Nat Biotechnol. 2010;28:511-5.

16. Guttman M, Garber M, Levin JZ, Donaghey J, Robinson J, Adiconis X, Fan L, Koziol MJ, Gnirke A, Nusbaum C. Ab initio reconstruction of cell typespecific transcriptomes in mouse reveals the conserved multi-exonic structure of lincRNAs. Nat Biotechnol. 2010;28:503-10.

17. Kim D, Langmead B, Salzberg SL. HISAT: a fast spliced aligner with low memory requirements. Nat Methods. 2015;12:357-60.

18. Dobin A, Davis CA, Schlesinger F, Drenkow J, Zaleski C, Jha S, Batut P, Chaisson M, Gingeras TR. STAR: ultrafast universal RNA-seq aligner. Bioinformatics. 2013;29:15-21.

19. Kim D, Pertea G, Trapnell C, Pimentel H, Kelley R, Salzberg SL. TopHat2 accurate alignment of transcriptomes in the presence of insertions, deletions and gene fusions. Genome Biol. 2013;14:R36.

20. Au KF, Jiang $H$, Lin $L$, Xing $Y$, Wong WH. Detection of splice junctions from paired-end RNA-seq data by SpliceMap. Nucleic Acids Res. 2010;38:4570-8.

21. Wang $K$, Singh D, Zeng Z, Coleman SJ, Huang Y, Savich GL, He X, Mieczkowski P, Grimm SA, Perou CM. MapSplice: accurate mapping of RNAseq reads for splice junction discovery. Nucleic Acids Res. 2010;38:e178.

22. Wu TD, Nacu S. Fast and SNP-tolerant detection of complex variants and splicing in short reads. Bioinformatics. 2010;26:873-81.

23. Liu J, Li G, Chang Z, Yu T, Liu B, McMullen R, Chen P, Huang X. BinPacker: packing-based de novo transcriptome assembly from RNA-seq data. PLoS Comput Biol. 2016;12:e1004772.

24. Chang Z, Li GJ, Liu JT, Zhang Y, Ashby C, Liu DL, Cramer CL, Huang XZ. Bridger: a new framework for de novo transcriptome assembly using RNAseq data. Genome Biol. 2015;16:30.

25. Peng Y, Leung HC, Yiu SM, Lv MJ, Zhu XG, Chin FY. IDBA-tran: a more robust de novo de Bruijn graph assembler for transcriptomes with uneven expression levels. Bioinformatics. 2013:29:i326-34.

26. Xie YL, Wu GX, Tang JB, Luo RB, Patterson J, Liu SL, Huang WH, He GZ, Gu SC, Li SK, et al. SOAPdenovo-Trans: de novo transcriptome assembly with short RNA-Seq reads. Bioinformatics. 2014;30:1660-6.

27. Simpson JT, Wong K, Jackman SD, Schein JE, Jones SJ, Birol I. ABySS: a parallel assembler for short read sequence data. Genome Res. 2009;19: 1117-23.

28. Schulz MH, Zerbino DR, Vingron M, Birney E. Oases: robust de novo RNAseq assembly across the dynamic range of expression levels. Bioinformatics. 2012;28:1086-92.
29. Kent WJ. BLAT-the BLAST-like alignment tool. Genome Res. 2002;12:656-64

30. Griebel T, Zacher B, Ribeca P, Raineri E, Lacroix V, Guigo R, Sammeth M. Modelling and simulating generic RNA-Seq experiments with the flux simulator. Nucleic Acids Res. 2012:40:10073-83.

31. Liu J, Yu T, Mu Z, Li G. TransLiG: a de novo transcriptome assembler that uses line graph iteration. Source Code SourceForge. 2019. https:// sourceforge.net/projects/transcriptomeassembly/files/ .

32. Liu J, Yu T, Mu Z, Li G. TransLiG: a de novo transcriptome assembler that uses line graph iteration. Source code. Zenodo. 2019. https://doi.org/10. 5281/zenodo.2576226

33. Liu J, Yu T, Mu Z, Li G. TransLiG: a de novo transcriptome assembler that uses line graph iteration. NCBI sequence read archive. 2019. https://www.ncbi.nlm.nih.gov/.

34. Liu J, Yu T, Mu Z, Li G. TransLiG: a de novo transcriptome assembler that uses line graph iteration. Simulation data. 2019. https://sourceforge.net/ projects/transassembly/files/TransLiG-Simulation-Data/.
Ready to submit your research? Choose BMC and benefit from:

- fast, convenient online submission

- thorough peer review by experienced researchers in your field

- rapid publication on acceptance

- support for research data, including large and complex data types

- gold Open Access which fosters wider collaboration and increased citations

- maximum visibility for your research: over $100 \mathrm{M}$ website views per year

At BMC, research is always in progress.

Learn more biomedcentral.com/submissions 